\title{
Mutmaßungen über den künftigen Kommunikationsbeitrag der Kirchen
}

von Ulrich Saxer

\section{Der Beitrag der Wissenschaft an die Zukunft der Kirchen}

„Keine Prognose ist harmlos"1, stellte Karl Jaspers bereits 1949 fest, und dies gilt auch weiterhin, sowohl für die Prognostiker wie für ihre Objekte. Zwar hat sich die Zukunftsforschung, nicht zuletzt unter der magischen Ausstrahlung der Jahreszahl 2000, allenthalben mächtig entfaltet und allein schon für den Bereich Technologie an die 100 verschiedene Prognosemethoden bzw. Methodenkombinationen entwickelt ${ }^{2}$, aber noch immer ist die Treffsicherheit zumal langfristiger, mehrere Sektoren umspannender Vorhersagen denkbar bescheiden, dermaßen in der Tat, daß ein Wissenschaftler, der sich auf dieses Tun einläßt, leicht in den Geruch des Unseriösen gerät. Ist dies immerhin bloß sein persönlicher Schaden, so können seine Prognosen, Zielformulierungen oder Planungshilfen sogar kollektives Unheil anrichten, wenn sie unangemessene Einstellungen und Verhaltensweisen veranlassen. Anders als die herkömmliche Grundlagenforschung die von ihr erklärten vergangenen oder gegenwärtigen Prozesse und Zustände beeinflußt die gesellschaftsbezogene Zukunftsforschung ihr Objekt regelmäßig im Sinne einer eigentümlichen Dialektik: Unheilsprognosen trachten danach, Bedingungen zu erwirken, die sie selber Lügen strafen, und Heilsprognosen suchen natürlich die diagnostizierten günstigen Trends zu verstärken ${ }^{3}$. An den Rezipienten zukunftsbezogener Aussagen, und somit auch dieses Aufsatzes, liegt es also nicht minder als an den Aussagen selber, wenn sie gute oder schlechte oder überhaupt keine Wirklichkeit werden.

Bei der Abschätzung des Kommunikationsbeitrags der Kirchen im Jahre 2000, wie hier erwartet wird, handelt es sich prognostisch sogar um eine derart schwierige Konstellation, daß "Mutmaßungen“ oder "educated guesses“, wie Johan Galtung schreibt", solche Bemühungen zutreffender charakterisieren als "Prognose" oder "Vorhersage". Irgendeinen Sinnbedarf, wie etwa denjenigen nach christlichen Gehalten, für eine mehr als 20 Jahre entfernte Zukunft vorhersagen zu wollen, hat mehr mit Prophetie als mit Prognose zu tun. Gerade für die langfristige Prognostizierung von Mentalitäten, die hier zu geschehen hätte, verfügt die Zukunftsforschung noch kaum über irgendwelche tauglichen Verfahren. Zudem sind die Kirchen in solcher vielfältiger Art und Weise als Kommunikationsinstitutionen tätig, daß es bereits die allergrößten Schwierigkeiten bereitet, nur schon ihrer gegenwärtigen Kommunikationsleistung gerecht $z \mathfrak{u}$ werden, geschweige denn die viel spätere Differenzierung dieses Kommunikationssystems vorauszusehen. Ferner werden auch die Kriterien erfolgreicher kirchlicher Kommunikation ebenso verschieden angesetzt wie der Religionsbegriff selber: Sie reichen vom bloßen Ansprechen bis zum BekehrenWollen und gestatten daher zumal dem Nichttheologen und Nicht-Kirchenmann auch

Prof. Dr. Ulrich Saxer ist amtierender Direktor des Publizistischen Seminars der Universität Zürich. 
keine nur halbwegs zuverlässige Zielprojektionen, die auf etwaige künftige Entwicklungen bezogen werden könnten ${ }^{5}$. Und schließlich sind Voraussagen bezüglich des gesellschaftlichen Kommunikationssystems dessen spezifischer Dynamik wegen mit besonders hohen Risiken behaftet ${ }^{6}$. Aus all diesen Gründen können die folgenden Ausführungen keinen höheren Verbindlichkeitsgrad als denjenigen von Vermutungen beanspruchen, und legitimiert sind sie in ihrer intuitiven Grundstruktur wie auch jede Delphi-Umfrage ${ }^{7}$ vor allem soweit, als sie auch wieder andere Leute zum Mitdenken und damit zum Mitgestalten statt zum bloßen Miterdulden der Zukunft anregen.

Dem dürften sich freilich einige Widerstände entgegenstellen, denn allem Anschein nach ist das Verhältnis von Religion bzw. Kirche und Zukunftsforschung besonders problematisch. Dies in erheblichem Unterschied zur kirchlich bereits viel intensiver rezipierten Kommunikationsforschung. Für Institutionen, die sich so sehr um das künftige Heil der Menschheit bemühen, hatten hingegen die Kirchen bis vor kurzem bemerkenswert wenig für die Zukunftsforschung übrig, neuerdings indes, unter dem Einfluß von Politisierungsschïben bzw. Bestandskrisen, mancherorts allerdings umso mehr'. Die längere Zeit überwiegend technologische Ausrichtung der Zukunftsforschung und ihre Tendenz, "status-quo-Prognosen "10 zu bevorzugen, d. h. künftige Systemzustände unter der Annahme weiterhin wirksamer Rahmenbedingungen und -tendenzen vorauszusagen, stehen eben in scharfem Gegensatz zur Vergangenheitsbindung, aber auch zur prophetischen Tradition der Kirchen, ihrem Denken und Wollen in umfassendsten Bezügen, wo Systemgrenzen wenig gelten ${ }^{11}$ und wo nach dem Willen Gottes zumindest grundsätzlich immer alles möglich ist. Die analytische Zukunftsforschung, um eine Theorie möglicher zukünftiger Systeme und Systemkonstellationen bzw. der Konsequenzen möglicher Entscheidungen bemüht und daher gerade an der Determinierung künftiger Systemzustände interessiert, hält sich dementsprechend mehrheitlich an das Jeremiaswort, beide, Propheten und Priester, seien Schälke ${ }^{12}$, und an die Maxime, der Sektor Religion sei für sie wenig lohnend, während die Kirchen wachsende Neigung für gesinnungsverträgliche Varianten der engagierten Zukunftsforschung mit ihrer charakteristischen Vermischung von Normativem und Faktischem bekunden ${ }^{13}$.

Für die Zukunft der Kirchen dürfte jedenfalls mehr und mehr von Belang werden, wieweit sie ihr eigenes Verhältnis zur Zukunftsforschung auf rationale Basis stellen und prognostische und ideologische Aktivitäten deutlicher scheiden werden.

In diesem Sinne sei auch der folgende Versuch selber noch etwas genauer charakterisiert. Den Ausgangspunkt dieser zukunftsbezogenen Aussagen bildet die Annahme, Kirchen, also der institutionalisierte Teil christlicher Religiositä $t^{14}$, seien in erster Linie Kommunikationsinstitutionen, Bedeutungsvermittlung also ihre Hauptfunktion und ihr gesellschaftliches Schicksal am stärksten an deren mehr oder minder erfolgreiche Erfüllung geknüpft. Insofern handelt es sich hier um kommunikationswissenschaftlich akzentuierte Zukunftsaussagen. Als Kriterium für den Funktionserfolg gilt das Ausmaß, in dem der kommunikative Beitrag der Kirchen die Probleme von gesellschaftlichen Systemen, von Kollektiven und Individuen lösen hilft. Über diesen Erfolg entscheidet die Art und Weise, in der sich die Kirchen als institutionalisierte Religiosität mit den kommunikationsrelevanten gesellschaftlichen Strukturen auseinandersetzen. Diese Strukturen wie Medienverhältnisse, Bevölkerungsmentalität etc. stellen einerseits Funktionsbedingungen für die kirchlichen Kommunikationsaktivitäten dar, andererseits bilden sie selber verfestigte Problemlösungen, dic durch 
neue wieder in Frage gestellt werden können. Prognostisch müßte daher auch die Dynamik dieser Strukturen mitberücksichtigt werden, was bei deren Vielfalt und mannigfachen gegenseitigen Abhängigkeiten hier eben nur im Sinne von Mutmaßungen und eines „surprise free future ${ }^{\text {"15 }}$ geschehen kann. Demgemäß wird im Folgenden der Unterschied zwischen internationalen und nationalen Strukturen weiterhin als entscheidend behandelt und als Gliederungsprinzip gewählt, wobei international der Akzent auf die weltpolitische und die technologische Entwidklungskonstellation gelegt wird, national, bei Beschränkung auf die entwickelten Demokratien, auf den institutionellen und mentalitätsmäßigen Aspekt.

\section{Der globale künftige Kommunikationsbeitrag der Kirchen}

\subsection{Kirchliche Kommunikation und weltpolitische Konstellation}

Weil die Kirche in ihren verschiedenen Ausprägungen eine weltumspannende Kommunikationsinstitution darstellt und auch im Jahre 2000 noch darstellen wird, müssen als äußerste Funktionsbedingungen zuerst die global relevanten Kommunikationsstrukturen und ihre Entwicklung skizziert werden. Den stärksten Bedingungszusammenhang für die kirchlichen Kommunikationsaktivitäten wird da wohl weiterhin die weltpolitische Konstellation bilden, die die Weltkommunikation in die Kommunikationssysteme entwickelter Demokratien, relativ entwickelter kommunistischer Staaten, kommunistischer und nichtkommunistischer Entwicklungsländer zerfallen läßt $t^{16}$. Der Trend zur Entwicklungsdiktatur dürfte unter diesen letzteren zunehmen, die Einheit des kommunistischen Lagers sich hingegen weiterhin zersetzen, aber auch die entwickelten Demokratien werden wohl ihre nationalen Besonderheiten kommunikativ nur unwesentlich unter dem Einfluß übernationaler Zusammenschlüsse abbauen.

Für die kommunizierende Kirche bedeutet dies vor allem, daß sie künftig in den Entwicklungsländern mit noch stärkerer Kontrolle rechnen muß. Da unter den Zielen der dortigen Eliten dasjenige des nation-building weit oben rangiert, werden christliche Missionierung, Verkündigung etc. vermehrt nur soweit toleriert werden, als sie mit diesen Zielen vereinbar sind. Es ist zu erwarten, daß insbesondere die protestantische Kirche solchen und andern Auflagen in erheblichem $\mathrm{Maß}$ entsprechen wird, unter Umformulierung spezifisch christlicher bzw. religiöser Ziele in solche der allgemeinen Entwicklungsförderung ${ }^{17}$. Die künftige innerkirchliche Willensbildung und die daraus resultierenden kommunikativen Zielsetzungen kommen hier schon als schwer prognostizierbare Größen ins Blickfeld.

Die Pluralisierung kommunistischer Machtzentren kann dagegen - braucht aber nicht - eine Erweiterung des dortigen Toleranzspielraums gegenüber kirchlicher Kommunikation mit sich bringen. Je nach den Bedürfnissen etwa des polnischen, ungarischen oder rumänischen Regimes wird diese Entwicklung jedenfalls recht unterschiedlich verlaufen. Auch dies wird vermutlich die Kirchen zur situativen Differenzierung ihrer Kommunikationsanstrengungen, unter Umständen auf Kosten mancher verbindlicher Gemeinsamkeiten, zwingen, mit dem Ergebnis vermehrter innerkirchlicher Spannungen bezüglich der zulässigen Interpretation der christlichen Botschaft und der optimalen christlichen Kommunikationsstrategie.

Der weiter bestehende, sich regional sogar verstärkende Partikularismus der entwickelten Demokratien wiederum hat zur Folge, daß nationalkirchliche Eigenheiten, 
z. B. der amerikanischen Kirchen bzw. derjenigen verschiedener europäischer Staaten, auch künftig und möglicherweise noch stärker die internationale kirchliche Kommunikation charakterisieren werden. Die international verschiedenartigen und auch widersprüchlichen Entwicklungstendenzen rufen eben allgemein nach einem gleidfalls verschiedenartigen und nicht selten widersprüchlichen globalen kirchlichen Kommunikationsbeitrag, auch innerhalb wie zwischen den Konfessionen, wollen die Kirchen überhaupt unter modernen Machtverhältnissen in größerem Maß als Kommunikationsinstitutionen zum Zuge kommen oder immerhin zugelassen werden. Immerhin dürften sich auch Tendenzen intensivieren, durch überkonfessionelle Einsätze und Strategien das Gewicht der kirchlichen Kommunikation international zu vergrößern.

\subsection{Kirchliche Kommunikation und sektorielle Entwicklung}

Eine Entwicklung, die besonders in Industriegesellschaften sich abzeichnet, aber im globalen Maßstab Modellcharakter gewinnen dürfte, ist diejenige in Richtung der sogenannten Informationsgesellschaft ${ }^{18}$, d. h. eines Gesellschaftstyps mit immer dominanterem Informationssektor. Der Tertiär- oder Dienstleistungssektor, dem herkömmlicherweise die Kommunikationsinstitutionen zurechnen, ist ja vielerorts selber bereits so umfassend und komplex geworden, daß er seinerseits intensivster kommunikativer Erschließung und Integration in die übrige Gesellschaft bedarf. Im Gefolge dieses gesellschaftsstrukturellen Bedarfs beginnt so etwas wie ein Quartärsektor ${ }^{10}$ für die Kommunikationsfunktion sich auszudifferenzieren, erkennbar ebenso an den sehr vermehrten öffentlichen kirchlichen Selbstexplikationen wie an den unerhört intensivierten Public-Relations-Bemühungen von Banken oder Verkehrsorganisationen.

Kommunikationsinstitutionen, wie auch die Vermehrung der Kommunikationsberufe und ihrer Träger ${ }^{20}$ verrät, gewinnen damit insgesamt vermehrt gesamtgesellschaftliche Bedeutung. Diesem für die künftige kirchliche Kommunikation günstigen Befund steht freilich der Umstand entgegen, daß um den grundsätzlich nicht allgemein vermehrbaren Wert "gesellschaftlicher Einfluß" sich auch immer mehr Kommunikationsinstitutionen streiten, neue spezialisierte Kommunikationsproduzenten und -vermittler, von der Werbung bis zu den verschiedensten Informationsdiensten, in großer Variationenvielfalt Rezipienten für die verschiedensten Botschaften suchen. Die jetzt schon von theologischer Seite vielfach schmerzlich empfundene Situation, daß die Kirche nur noch Kommunikationsanbieter unter vielen andern, zum Teil schon erheblich besser organisierten, ist und daß die propaganda fidei mit politisch und wirtschaftlich viel mächtigeren Arten der Propaganda konkurrieren muß, wird sich also durchaus noch allenthalben verschärfen.

Man könnte sich sogar fragen, ob die Kirchen nicht gut daran täten, noch vermehrt Schwerpunkte im nicht primär kommunikativen Funktionsbereich, etwa der Caritas oder der Kranken- und Altersfürsorge, zu setzen, wo die gesellschaftlichen Defizite offenkundiger und ein subsidiärer Gestaltungsbeitrag der Kirchen intensiver gewünscht und empfunden und als Propaganda der Tat auch wieder die kirchliche Kommunikationssituation verbessern würde. Als Kommunikationsinstitution unter andern Kommunikationsinstitutionen wird ja die Kirche, zum Teil mit kommunikations- und sozialwissenschaftlicher Hilfe $\mathrm{e}^{21}$, immer konsequenter deren Kommunikationsstrategien auffangen, erfolgreiche kopieren müssen etc., mit dem Resultat, daß die kirchliche Rhetorik derjenigen der allgemeinen Public Relations inskünftig 
ähnlicher und ähnlicher, die kirchliche Selbstdarstellung Konzessionen an die verschiedensten Kommunikationsmoden, Aktualitäten und populären Schlagworte, etwa demjenigen von der "Transparenz ${ }^{\text {"22 }}$, aus außerreligiösen Kommunikationsbereichen machen wird. Dies beschwört auch in den kommenden Jahren immer wieder innerkirchliche Spannungen herauf, da Teile des Klerus wie der Kommunikationsadressaten die Reinheit der Lehre oder zumindest die Unverwechselbarkeit des christlichkirchlichen Sprechens vermissen, und zugleich leiden darunter natürlich der unüberhörbare kirchliche Anspruch auf kommunikationsethische Vorbildlichkeit und die kirchlichen Bemühungen um die Entwicklung einer allgemeinverbindlichen Kommunikationsethik im Sinne eines ethischen Steuerungsversuchs des neuen Quartärsektors.

Die sektorielle Verstärkung des Kommunikationswesens hat aber auch zur Folge, daß der Kommunikationstechnologie fortdauernd hohe gesellschaftliche Bedeutung zukommen und sie sich dementsprechend weiterentwickeln wird. Die Chancen der kirchlichen Kommunikationsanstrengungen liegen diesbezüglich in den Entwicklungsländern und in den industrialisierten Gesellschaften unterschiedlich; sie könnten aber auf jeden Fall noch wirkungsvoller als bis anhin genutzt werden. Soweit die politische Konstellation in den Entwicklungsländern es zuläßt, wären hier vermehrt Investitionen in preiswerte Kommunikationstechnologien denkbar, neben illustrierten Druckmedien vor allem Radio, wobei außer dem Betrieb kircheneigener oder immerhin kirchlich unterstützter Sender auch kollektive Empfangssysteme zum Zwecke der Diskussion und der adäquaten Rezeption des Ausgestrahlten zu organisieren sind ${ }^{23}$. Die Schwäche der dortigen Kommunikationsinfrastrukturen ruft geradezu nach solchen infrastrukturellen kirchlichen Kommunikationshilfen, und sei es auch nur in Form technologisch kompetenter Kommunikationsspezialisten.

Anders in den industrialisierten Ländern, die allesamt über sehr stark entfaltete Mediensysteme verfügen, hingegen zunehmend Defizite in der interpersonalen Kommunikation erkennen lassen. Die Befunde der deutschen Kommission für den Ausbau des technischen Kommunikationssystems $(\mathrm{KtK})$, die stärkeren Bedarf nach verbesserter Telefonversorgung als nach Kabelfernsehen und eine besonders hohe Wertschätzung und Leistungsfähigkeit der Kommunikationsform des Gesprächs erwiesen haben $^{24}$ - darin zweifellos über die Bundesrepublik Deutschland hinaus für die industriegesellschaftlichen Verhältnisse allgemein in vielem repräsentativ -, lassen jedenfalls verstärkte Telefonseelsorge, kommunikative Direktkontakte als dringlichere und daher auch mehr Erfolg verheißende kirchliche Kommunikationsbemühungen erscheinen denn weitere wirtschaftliche und politische kirchliche Kraftakte im Radio-, Fernseh- oder Kabelbereich. Eigene Sender zumal werden auch künftig in den entwickelten Demokratien die kirchlichen Kommunikationsanstrengungen eher gettoisieren als multiplizieren, wenn auch der institutionelle Repräsentationsgehalt solcher Sender, aber auch kirchlicher Printmedien, nicht bagatellisiert werden soll. Freilich ist anzunehmen, daß die Kirchen im Bestreben, modern zu sein und kommunikativ mithalten zu können, auch diese Wiederaufwertung ihrer ursprünglichen Kommunikationsmöglichkeiten erst mit einiger Verspätung wahrnehmen werden, scheint es doch $\mathrm{zu}$ den allgemeinen Eigentümlichkeiten kirchlicher Kommunikationsstrategie im späteren 20. Jahrhundert zu gehören, außerreligiöse Kommunikationsentwicklungen irgendeinmal nachzuholen und darüber spezifische eigene Kommunikationschancen $\mathrm{zu}$ vernachlässigen ${ }^{25}$. 


\section{Der künftige kirchliche Kommunikationsbeitrag in den entwickelten Demokratien}

\subsection{Kirchen und andere Kommunikationsinstitutionen}

Wenn nun im Folgenden das Gesichtsfeld auf die entwickelten Demokratien eingeschränkt wird, dann in erster Linie, damit noch konkretere Mutmaßungen über den künftigen kirchlichen Kommunikationsbeitrag angestellt werden können und dabei der widersprüchlichen Vielfalt der Entwidklungstendenzen noch besser Rechnung getragen werden kann. Schon die Unterschiede $z$ wischen protestantischer und katholischer Kirche sind und bleiben ja so beträchtlich, daß es nur bedingt statthaft ist, sie überhaupt als identische Prognoseobjekte zu behandeln. Andererseits findet sich mutatis mutandis die Spannung der Kirche zu den übrigen Kommunikationsinstitutionen natürlich auch in andern Gesellschaften als bloß den entwickelten Demokratien, und ebenso wären dort Mentalitätsveränderungen für weitere Vorhersagen zu berücksichtigen.

Für die Stellung und das Wirken der Kirche unter den andern Kommunikationsinstitutionen ist vor allem bedeutsam, daß sie hinsichtlich der Vielfalt von Kommunikationsbemühungen nach wie vor alle andern großen Kommunikationsinstitutionen übertrifft. Wirtschaftliche und politische Kommunikationsinstitutionen, sei es von Verbänden, Parteien oder staatlichen Behörden, bleiben ja thematisch auf die Systemprobleme von Wirtschaft und Politik beschränkt, und unter den kulturellen Kommunikationsinstitutionen, denen die Kirchen primär zurechnen, begnügt sidh etwa auch die Schule durchaus mit erzieherischen Gehalten. Aber auch das unerhört expansive Kommunikationssystem der Wissenschaft mutet im Vergleich zu den Kirchen überaus restringiert an; zwar thematisiert sie immer weitere Daseinsbereiche, äußert sich aber über diese gemäß bestimmten Verläßlichkeitsregeln nur in einer hochnormierten Form, ganz anders als etwa der Protestantismus mit seiner Vielfalt von Sprechweisen. Selbst die aktuell-universellen Medien Presse und Rundfunk fallen insofern gegenüber den Kirchen $a b$, als sie über keine interpersonalen Kommunikationsorganisationen wie jene in den Pfarreien verfügen ${ }^{26}$. Die Zukunft der Kirchen hängt unbedingt von der Meisterung der Probleme $a b$, die dieses extrem vielfältige kommunikative Engagement mit sich bringt.

Einzig der Kulturbereich Kunst weist in der Moderne eine ähnliche Vielfalt von Kommunikationsweisen nach Themen, Formen, Medien und Adressaten auf - eine Parallele, die auch für die Zukunft der kirchlichen Kommunikation einigen Aufschluß verspricht. Die moderne Kunst scheint unter der Fülle der als „Kunst“ tolerierten Kommunikationsmöglichkeiten an kultureller Identität einzubüßen; hier und heute definiert sich das ursprünglich ästhetische Kommunizieren als „Wissenschaft ${ }^{*}$, dort und dann als „Politik", Wechsel, die durchaus an diejenigen des ursprünglich religiösen Kommunizierens gemahnen. Gerade die unverkennbare Schwächung kirchlicher Kunst verrät den Abbau der institutionellen Verbindlichkeit von Kunst bei aller künstlerischen Massenproduktivität in den entwickelten Demokratien, so wie die fast beliebige Vermehrung neuer kirchlicher Kultformen den kulturellen Identitäts- und institutionellen Verbindlichkeitszerfall der Kirchen ${ }^{27}$. Diese Entwicklung wird sich solange fortsetzen, wie das gegenwärtige kommunikative Selbstverständnis der Kirchen fortdauert. Unter dem Einfluß weiterer Strukturveränderungen, aber auch zusätzlich legitimiert durch das fast beliebig weite kirchliche und künstlerische Kommunikationsspektrum, stellen denn auch bereits vielerorts irgendwelche Beteiligungsnormen an der religiösen Kultur ebensowenig allgemeine Voraussetzungen für 
höhern Status dar wie literarische oder künstlerische Bildung. Freilich ist nicht zu übersehen, daß die katholische Kirche immerhin noch aus einem im weitesten einheitlichen Sinnhorizont heraus kommuniziert, während die kulturelle Identität der protestantischen Kirche dermaßen undeutlich geworden ist, daß sie als Ganze keinen wirklich als "protestantisch" mehr identifizierbaren und damit spezifischen Kommunikationsbeitrag leistet, der die institutionelle Verbindlichkeit dieses Sprechens vor weiterer Beeinträchtigung bewahrte.

Aus der Vielfalt ihrer kommunikativen Anstrengungen resultiert also nach dieser Analyse das elementarste Gegenwarts- und Zukunftsproblem der christlichen Kirchen, dasjenige ihrer kulturellen Identität nämlich. Identitätsbewahrung ist für jedes gesellschaftliche System eines der vier von ihm zu meisternden Fundamentalprobleme, neben Integration, Umweltanpassung und Zieldurchsetzung für eine Kulturinstitution wohl das wichtigste, und wie spannungsvoll da die Problemlösungen zueinander stehen können, veranschaulicht gerade die Kommunikationsinstitution Kirche exemplarisch. Sie kann ja gegebenenfalls recht erfolgreich das Ziel durchsetzen, möglichst viele Leute kommunikativ zu erreichen, aber eben um den Preis substantieller Identitätsverluste: Wo alles Erdenkliche plötzlich als "christlich“ gilt oder der Attraktivität der Botschaft deren sämtliche inhärenten Widerstände aufgeopfert werden, da bleibt die Kirche, wie so oft gefordert, zwar „im Gespräch“, aber letztlich einem fremdgesteuerten. Wohl gelingt so eine gewisse Anpassung an die säkularisierte Umwelt, indes auf Kosten der spezifischen Funktion der Institution Kirche und meist auch ihrer Integration, da die Strenggläubigen gegen solche Identitätspreisgaben aufbegehren. Umgekehrt riskiert natürlich die mit einem übergreifenden Lehr- und Autoritätsanspruch in der pluralistischen und säkularisierten Demokratie auftretende Kirche die Verweigerung der andern Kommunikationsinstitutionen und aller nicht schon kirchlich Autoritätsgläubigen ${ }^{28}$ zu provozieren. Das durchsetzbare Ziel kann dann auf jeden Fall nicht mehr dauernde Massenevangelisation heißen.

Diese und weitere Spannungen lassen sich als Ausdruck struktureller Gegensätze innerhalb der Kirchen interpretieren, die auch künftig zu schweren Ziel-/Mittel- und Zentrums-/Parochiekonflikten führen werden. Als Hauptgegensatz kann wohl derjenige zwischen Glaubenstradition und Kommunikationsorganisation der Kirchen bezeichnet werden. Dem abnehmenden allgemeinen Interesse an dieser Tradition und den auf sie gestützten Deutungs- und Erklärungsschemata steht eine weiterhin auf Massen- wie Individualkommunikation angelegte, teilweise sogar expandierende Großorganisation gegenüber, die unter diesen Umständen kommunikative Zielkorrekturen geradezu erzwingen wird, nämlich in Richtung noch stärkerer situativer Anpassung der verschiedenen Elemente dieser Kommunikationsorganisation. $\mathrm{Zu}$ einem innerkirchlich allgemein anerkannten Zielsystem wird man denn auch in näherer Zukunft keineswegs gelangen; kirchliche Zentralen werden vielmehr mit wachsenden Schwierigkeiten zu kämpfen haben, den verschiedenen kirchlichen Teilsystemen gegenüber - die in der Soziologie nicht zufälligerweise bei einer gewissen Eigenständigkeit allgemein "parochiale" genannt werden ${ }^{29}$ - vereinheitlichende kommunikative Direktiven durchzusetzen, und die kirchlichen Sprecher der mittleren und unteren Ränge werden weiterhin zwischen Erfolgszwang und Gehorsamspflicht lavieren.

Hinsichtlich der verschiedenen Kommunikationsformen, unter denen die Kirchen wirksam werden, sind die Folgen dieser strukturellen Spannungen allerdings unter- 
schiedlich. Neben der fachtheologischen Weiterentwicklung der kirchlichen Glaubenstradition als Basis aller weiteren Kommunikationsformen und neben der Organisationskommunikation, die die Teile der Kirche gemäß diesen theologischen Erkenntnissen koordinieren und die Repräsentation der Kirchen gegenüber ,außen “ besorgen muß, gibt es ja außer dem Kult u. a. die erzieherische, die seelsorgerische und die institutionskontrollierende Kirchenkommunikation. Unter die letztere fallen alle jene Aktivitäten, mit denen die Kirchen weiterhin das politische, wirtschaftliche, soziale und kulturelle Geschehen auf institutioneller Ebene zu steuern versuchen, indem sie bestimmte Parteien begünstigen, Behörden kritisieren, Werbepraktiken anprangern, Minoritätenbelange artikulieren etc. Mit diesen Kontrollansprüchen konkurrieren die Kirchen am eindeutigsten die Kommunikationsinstitutionen dieser Bereiche, aber natürlich auch diejenigen der Erziehung, sobald etwa das Prinzip der Konfessionsschule gegen dasjenige der Staatsschule forciert wird. Besonders offenkundig ist dieses Konkurrenzverhältnis auch in den Massenmedien, wo die Kirchen vielfach eigentliche Berufsmedienpolitiker ins Feld schicken, um ihre Anteile an Einfluß über den Rundfunk einzutreiben ${ }^{30}$.

Für die Zukunft ist allerdings anzunehmen, daß noch vorhandene Privilegien der Kirchen in diesem Konkurrenzkampf vielerorts abgebaut werden, u. a. dem Ruf nach vermehrter Trennung von Kirche und Staat gemäß31. Dann wird auch der Rückgang ihrer wirtschaftlichen Mittel den kommunikativen Einsatz der Kirchen drastisch reduzieren. Jedenfalls liegen solche Entwicklungen auf der Linie der fortschreitenden Differenzierung der modernen Gesellschaften in funktionale Subsysteme, in denen bestimmte Leistungen für die Gesamtgesellschaft spezialisiert sind. Das hier für den künftigen kirchlichen Kommunikationsbeitrag wichtigste Stichwort lautet darum: Kompetenz. Nur wenn die Kirchen in kompetenter Form sich in die politische oder die Wirtschaftskommunikation einschalten, fällt ihr entsprechender Beitrag überhaupt ins Gewicht, und die Beurteilungsstandards werden in diesen Bereichen nicht von den Kirchenvertretern beschlossen und durchgesetzt. Es bestehen begründete $Z_{w}$ eifel, daß die Kirchenkommunikation in absehbarer Zeit den für solche Gebiete erforderlichen Spezialisierungs- und Kompetenzgrad erreichen wird.

Eher wahrscheinlich ist, daß die Kirchen weiterhin vor allem traditionelle moralische Normen in den verschiedenen institutionellen Bereichen sanktionieren ${ }^{32}$ und damit auch innerhalb ihrer angestammten Kompetenzsphäre bleiben werden. Und diese ist nun einmal diejenige der Theologie! Deren Differenzierung hat freilich einen Grad erreicht, daß diese spezialwissenschaftliche Offentlichkeit je länger desto weniger mit der Gesamtöffentlichkeit zusammenfällt, der kirchlichen Organisationskommunikation also immer schwierigere Aufgaben der kirchlichen Selbstexplikation gegen innen und außen aufgebürdet werden. Die Misere des Wissenschaftsjournalismus ist eben auch diejenige der theologischen Massenkommunikation, und diese Parallele wird auch in Zukunft gelten. Dies aber heißt: Die verschiedenen Teiltheologien, die für die unterschiedlichsten Daseinsbereiche entwickelt werden, werden auch in Zukunft ebensowenig zusammenfinden wie vielfach Theologie und kirchliche Selbstrepräsentation in den Aussagen ihrer verschiedenen Amtsträger. Der prekäre Zusammenhang $\mathrm{zwischen}$ Theologie und kirchlicher Organisationskommunikation schwächt denn auch die Legitimationsbasis des kirchlichen Sprechens überhaupt.

Theologie wird unter diesen Umständen weiterhin und noch verstärkt Spezialwissen für Spezialisten bleiben, da nicht einzusehen ist, welche kirchlichen Kommunikationsstrukturen die rasche und adäquate Umsetzung dieser Erkenntnisse für 
die Allgemeinheit besorgen könnten. Im Funktionsbereich der Erziehung werden demnach wie eh und je neben weltlichen Instruktionsstätten, die hinter dem neuesten Stand der Wissenschaft nachhinken, kirchliche Instruktionsstätten, die den gestrigen Stand der Theologie vertreten, die Jugendlichen belehren. Und die Seelsorge desgleichen wird weiterhin höchst unterschiedliche Anleihen bei Psychologie und Sozialpädagogik mit verschiedenen theologischen Substraten verbinden - nicht unbedingt zum Schaden der seelsorgerischen Kommunikation, die ja ein hohes Maß an situativer Individualisierung verlangt, aber mit dem Resultat, daß ehemals einigermaßen klare Erwartungen des Publikums an die Rolle des Seelsorgers ${ }^{33}$ immer konturloser oder widersprüchlicher werden und damit auch schwerer zu befriedigen sind.

\subsection{Kirchen und Mentalitätswandel}

Vom institutionellen Kommunikationsangebot der Kirchen her ist damit die Rede noch auf den kollektiven und individuellen Bedarf nach diesem gekommen. So wie es allmählich Schwierigkeiten bereitet, das Spezifische des kirchlichen Sprechens auszumachen, so fällt auch die Ermittlung von Intensität und Art der Nachfrage nach kirchlicher Kommunikation bereits in der Gegenwart schwer, geschweige denn für die Zukunft. Wenn selbst der Verallgemeinerungsgrad der Säkularisierungsthese nach wie vor umstritten ist ${ }^{34}$, dann läßt sich erst recht wenig Sicheres über die künftige Empfänglichkeit der Bevölkerungen entwickelter Demokratien für kirchliche Kommunikation vorhersagen, außer daß die mannigfaltigsten Konstellationen von Sozialstrukturen die entsprechenden Mentalitäten in den verschiedenen Ländern recht unterschiedlich beeinflussen werden. In katholischen Ländern mit einem traditionell großen kirchlichen Beitrag zum Bildungswesen z. B. wird das soziale Fortkommen auch künftig stärker von der Rezeption kirchlich vermittelter Gehalte abhängen und diese dementsprechend auch größer bleiben als in protestantischen Ländern mit praktisch reinen Staatsschulen. Ebenfalls kann selbstverständlich auch weiterhin mit verbreiteten religiösen Bedürfnissen, $d . h$. solchen nach umfassenden Sinndeutungen der Existenz mit Transzendenzbezug und nach verbindlicher Orientierung und geistiger Hilfe in Existenzkrisen etc. gerechnet werden; wie weit aber diese Bedürfnisse sich gerade auf Dogmatiken ${ }^{35}$ richten werden, die um die Begriffe Gott, Glauben, Kirche, Auferstehung, Offenbarung und überhaupt um das christliche Erbe zentriert sind, ist eine ganz andere Frage.

Die Indikatoren "Teilnahme am kirchlichen Kult" und "Teilnahme an religiöser publizistischer Kommunikation " weisen jedenfalls mehrheitlich und seit längerem praktizierende Christen und Publika religiöser Publizistik als Bevölkerungsminderheiten aus $s^{38}$. Es ist unter der Annahme einer surprise free future nicht einzusehen, wie und warum sich dies in den pluralistischen Demokratien in Zukunft ändern soll. Bei geschwächter Sanktionierbarkeit kirchlicher Autoritätsansprüche, verunklärten oder verblaßten Erwartungen an die Kirchen und vielfältigen, attraktiven andern Kommunikationsangeboten ist in absehbarer Zeit kein allgemeiner Mentalitätswandel im Sinne irgendeiner vertieften allgemeinen Rechristianisierung zu gewärtigen, entsprechende Moden, Meinungs- und kurzlebige Verhaltenswellen also, hingegen durchaus. Damit ist freilich noch nichts über den möglichen Wandel des christlichen Kommunikationsangebots gesagt, der natürlich auch wieder die Nachfrage mitsteuert. Hier wirken sich nun alle unter 3.1 skizzierten Tendenzen aus, die in Einbußen an Geschlossenheit und Spezifität der kirchlichen Kommunikation gipfeln. Dem ist noch hinzuzufügen, daß die Mobilität der Bevölkerung und die wachsende 
Internationalisierung der Massenkommunikation das Aufkommen religiöser und religionsähnlicher Mischformen noch weiter begünstigen werden. Die Angleichung der Kirchenkommunikation an außerkirchliche Kommunikationsformen und -instanzen wird sich denn auch weiter verstärken. Sie findet freilich ihre Schranken am kirchlichen Anspruch, allgemeinverbindlich zu kommunizieren, das Ganze zu verantworten. Gerade dies schreckt zweifellos viele Rezipienten der Massenkommunikation ab und läßt sie auch in Zukunft andere Medien-Freizeitofferten wählen; es gerät dieser universelle Kommunikationsanspruch aber auch in Widerstreit mit andern Kommunikationsinstitutionen, sobald dadurch deren partikuläre Kommunikationsintentionen und -muster in Frage gestellt werden. Dies heißt keineswegs, daß darum z. B. kirchliche Kritik im Namen ethischer Prinzipien an Herrschafts- und Geschäftspraktiken funktionslos sei. Es bleibt aber ungewiß, wieweit der kirchliche Anspruch auf universelle Sinngebung bei den inneren Widersprüchen der Kirchen und unter dem Eindruck der allgemeinen gesellschaftlichen Arbeitsteilung einer gewandelten Mentalität überhaupt noch als legitim erscheinen kann.

Für den Bedarf nach kirchlichen Kommunikationsleistungen gegenwärtig wie künftig bestimmend und bezeichnend ist jedenfalls die Differenzierung der nominell christlichen Bevölkerung in Intensiv- und Reduktionssegmente ${ }^{37}$. Die daraus resultierenden Gegensätze von Aktivisten und gelegentlichen Nutznießern werden die kirchlichen Amtsträger auch in Zukunft ständig vor heikle Zielkonflikte stellen, die sich in zahlreichen Widersprüchen zwischen den Konzeptionen der Volks- und der Minoritätenkirche niederschlagen. Der ausgesprochen selektive Bedarf der Bevölkerungsmehrheit nach kirchlicher Kommunikation, gewissen Kultleistungen zumal ${ }^{38}$, wird es den Kirchen jedenfalls immer mehr erschweren, diese Mehrheit nicht bloß mit einigen Dienstleistungen, sondern als verkündigende zu erreichen. Der subsidiär Defizite der mitmenschlichen und sozialen Fürsorge ausgleichenden Kirche ist denn auch eine günstigere Prognose auszustellen als der für eine bestimmte Glaubenstradition werbenden. In diesem Sinne dürften sogar erhebliche Chancen für die Kirchen im Bereich der lokalen Vergemeinschaftung, im Aufbau kleinräumiger Solidaritätssysteme liegen, nach denen, gewissermaßen kompensatorisch zu den gesellschaftlichen Gesamttrends, offenbar wieder stärkere Nachfrage besteht.

Wieweit die Kirchen diese sozusagen traditionellen Aufgabenbereiche wieder aufwerten werden, hängt allerdings ebensosehr wie die etwaige Präzisierung und Konkretisierung der christlichen Botschaften auf Kosten ihrer universellen Verwendbarkeit von der Entwicklung des kirchlichen Selbstverständnisses ab. Alle gesellschaftlichen Subsysteme müssen ihre Funktionen spezifizieren und ihre Grenzen irgendwie definieren ${ }^{99}$, und diese Notwendigkeit wird gerade den Kirchen immer stärker zu schaffen machen, weil sie bis jetzt diese Akte der Selbstbegrenzung erst ungenügend vorgenommen haben ${ }^{40}$. Wenn die Kirchen an die Gestaltung der Gesellschaft wirklich funktionale Beiträge leisten wollen, dann müssen sie sich in der Gesellschaft auch orten lassen und nicht letztlich immer noch den Anspruch erheben, überall sei Kirche. Zwar scheint der Gehalt der christlichen Botschaft derart reich und vielfältig interpretierbar zu sein, daß er die Selbstdefinition der Kirchen als Alternativen zum Gegebenen, als subsidiäre Dienstleistungsorganisationen oder als autoritäre Steuerungsinstanzen gestattet, aber eben um den Preis eines diffusen Leistungsprofils und nicht selten der Verkennung primärer Bedarfslagen. Wenn z. B. Religion als Einstellungsdimension sich tatsächlich um Schutzbedürftigkeit, Transzendieren, Orientierungswillen, Teilnahme an kultischen Veranstaltungen und konser- 
vative Gefühlslage konzentriert ${ }^{41}$, dann wird eben der kirchliche Kommunikationsbeitrag dieser Bedürfnislage entsprechen müssen, soll er nicht wie so of heute schon Angebot ohne Nachfrage bleiben.

\section{Anmerkungen:}

1. Zit. nach Scholder, Klaus: Grenzen der Zukunft. Stuttgart-Berlin-Köln-Mainz 1973. S. 79.

2. Jantsch, Erich: Technological Forecasting in Perspective. Paris 1967.

3. Merton, Robert K.: Die Eigendynamik gesellschaftlicher Voraussagen. In: Topitsch, Ernst (Hrsg.): Logik der Sozialwissenschaften. Köln-Berlin 1965.

4. Ornauer, Helmut / Wiberg, Hakan / Sicinski, Andrej / Galtung, Johan (Hrsg.): Images of the world in the year 2000. The Hague-Paris 1976. S. 20.

5. Exemplarisch für solche Widersprüche sind etwa die Sammelbände von Schatz, Oskar (Hrsg.): Hat die Religion Zukunft? Graz-Wien-Köln 1971, und von Wössner, Jakobus (Hrsg.): Religion im Umbruch. Stuttgart 1972.

6. Vgl. z. B. Magnus, Uwe (Hrsg.): Massenmedien in der Prognose. Berlin 1974, und Müller-Neuhof, Klaus: Kommunikationspolitik und Kommunikationsprognose. Stuttgart 1974.

7. Linstone, Harold A. / Turoff, Murray (Hrsg.): The Delphi Method. London-Amsterdam 1975.

8. Vgl. z. B. Koller, Erwin: Christliche Botschaften unter den Bedingungen der Fernsehkommunikation. Diss. Fribourg 1977.

9. Hierfür zeugt etwa die vom Schweizerischen Pastoralsoziologischen Institut unternommene Prospektivstudie „Kirche 1985“. St. Gallen 1970.

10. Tonnemacher, Jan: Prognosen für Massenmedien als Grundlage der Kommunikationspolitik in der BRD. Diss. Berlin 1975. S. 6.

11. Charakteristisch etwa Schweizerisches Pastoralsoziologisches Institut: a.a.O. Abschnitt 8.

12. Jeremia 23, 11.

13. Häufig genannte Autoritäten sind etwa Ossip Flechtheim, Robert Jungk, Georg Picht. Vgl. auch Schatz, Oskar (Hrsg.): a.a.O. S. 299 f.

14. Matthes, Joachim: Kirche und Gesellschaft. Hamburg 1968. S. 245.

15. Der Begriff stammt von Hermann Kahn und wird von Oskar Schatz bezeichnenderweise kritisiert (a.a.O. S. 18).

16. Vgl. Frey, Frederick W.: Communication and Development. In: de Sola Pool, Ithiel / Schramm, Wilbur et al. (Hrsg.): Handbook of Communication. Chicago 1973. S. 356; Unesco : World Communications. Paris 1975

17. Dies entspricht dem von Gerhard Schmidtchen diagnostizierten "protestantischen Syndrom" (Schmidtchen, Gerhard: Gibt es eine protestantische Persönlichkeit? Zürich 1969, S. 33 f.).

18. Arbeitsgemeinschaft für Kommunikationsforschung: Telekommunikationsbedarf. Literaturanalyse. München 1975. S. 101 f.; Bell, Daniel: Die nachindustrielle Gesellschaft. Frankfurt-New York 1975.

19. Kahn, Hermann / Wiener, Anthony J.: Ihr werdet es erleben. Voraussagen der Wissenschaft bis zum Jahre 2000. Wien 1968.

20. Parker, Edwin, B.: Social Implications of Computer/Telecommunications System. Paris 1975.

21. Vgl. als Beispiele unter vielen Albrecht, Horst: Kirche im Fernsehen, Massenkommunikationsforschung am Beispiel der Sendereihe „Das Wort zum Sonntag“. Hamburg 1974, und Koller, Erwin: a.a.O.

22. Vgl. u. a. Schweizerisches Pastoralsoziologisches Institut: a.a.O. Abschnitt 62.

23. Solche Rezeptionshilfen haben sich vielfach in den Entwicklungsländern als unerläßlich erwiesen.

24. Kommission für den Ausbau des technischen Kommunikationssystems: Telekommunikationsbericht und 8 Anlagebände. Bonn 1976.

25. Vgl. u. a. Koller, Erwin: a.a.O., und Saxer, Ulrich: Massenkommunikation als Mittel christlicher Verkündigung. In: „Communicatio Socialis" 2:1969, H. 2. 
26. Jene aber durchaus schon über eigene Informations- und Agenturdienste, um auch noch die publizistischen Medien im Sinne der kirchlichen Informationsinteressen $\mathrm{zu}$ beliefern.

27. Dieser schlägt sich beispielsweise auch in der Massenpublizistik nieder. (Vgl. Dörger, HansJoachim: Religion als Thema. Hamburg 1973. S. 395 f.).

28. Dies entspricht der aus der Wirkungsforschung bestbekannten Tendenz zur Selbstbestätigung durch Kommunikation.

29. Etzioni, Amitai: Die aktive Gesellschaft. Opladen 1975. S. 560.

30. Entsprechend versierte Kleriker werden ja eigens für solche $Z$ wecke freigestellt.

31. Uber eine entsprechende Initiative muß gegenwärtig in der Schweiz befunden werden.

32. Berger, Peter L.: The Noise of Solemn Assemblies. New York 1961. S. 40/1.

33. Deschwanden, Leo von: Eine Rollenanalyse des katholischen Pfarreipriesters. In: Matthes, Joachim (Hrsg.): Beiträge zur religionssoziologischen Forschung. Internationales Jahrbuch für Religionssoziologie Bd. IV. Köln - Opladen 1968.

34. Baum, Gregory: Der Fortbestand des Sacrum. In: „Concilium“ 9. Jg. (1973) H. 1, S. 3

35. Luhmann, Niklas: Religion als System. Thesen. In: Dahm, Karl Wilhelm / Luhmann, Niklas / Stoodt, Dieter: Religion-System und Sozialisation. Darmstadt-Neuwied 1972. S. 12.

36. Vgl. u. a. Holl, Adolf: Prognosen für den sozialen Stellenwert religiöser Phänomene. In: Wössner, Jakobus (Hrsg.): a.a.O. S. 52; Koller, Erwin: a.a.O. S. 181 f. Entsprechend bescheiden ist auch der Platz- und Programmanteil religiöser Themen in Presse und Rundfunk; im deutschen und schweizerischen Fernsehen z. B. machen religiöse Sendungen keine $2 \%$ aus.

37. Holl, Adolf: a.a.O. S. 52.

38. Vgl. Anmerkung 37.

39. Luhmann, Niklas: Die Organisierbarkeit von Religionen und Kirchen. In: Wössner, Jakobus (Hrsg.): a.a.O.

40. Bezeichnend sind etwa die Verlegenheiten bei der Prämiierung christlicher Medienbeiträge. (Vgl. epd/Kirche und Rundfunk Nr. 39 vom 25. Mai 1977: Wo endet „religiöse Thematik“?)

41. Holl, Adolf: a.a.O. S. 47.

\section{S U M M A R Y}

The criterion of the Church's success in its communicative function will be the extent to which she helps solve the problems of society and of the individual. In this task, the Church in the year 2000, as a worldwide communications institution, will also be subject to the then existing global communications systems. These will consist of the communications of developed democracies, of relatively developed communist states, and of the communist and noncommunist developing countries. The trend towards a development dictatorship in developing countries is likely to continue, and the unity of the communist camp is likely to become more and more dispersed. In all probability the developed democracies will not sacrifice much of their national characteristics to supra-national institutions. In industrial societies particularly, the role of information tends to change from that of a service to an independent institution. This can be seen in the increasing number of communications professions. On this account, more and more communications institutions have entered the struggle to gain a hearing with a public which is necessarily limited. In spite of the opportunities now opening for the Church, this competition also affects her situation. The Church has a plurality of means of communication over and above all other institutions, which are often limited by business, political or cultural factors. One of the most important present and future problems of the Christian Churches is to keep their own identity. Adaptation to the secular environment can mean a loss of the true function and integrity of the Church. The increasing division between active members and nominal Christians is a problem to be faced, as it is a source of conflict. The Churches have to specify their functions and define their limits. 


\section{RÉSUMÉ}

La mesure dans laquelle la collaboration communicative de l'Eglise aide à résoudre les problèmes des structures sociales des groupes et des individus a valeur de critère de succès dans sa fonction en tant qu'institution de communication. En cela décident, lors d'une situation de communication à l'échelon mondial et à laquelle l'Eglise sera également témoin en l'an 2000, les structures de communication globalement essentielles et leurs développements, c'est-à-dire les systèmes de communication des démocraties développées, les états communistes relativement développés, les pays communistes et non-communistes du tiersmonde. Le mouvement vers une dictature du développement devrait, en même temps, augmenter chez ces derniers, l'unité du camp communiste continuer à se décomposer, et même les démocraties développées réduire leurs particularismes nationaux bien qu'uniquement accessoirement sous l'influence des unions supranationales. Particulièrement dans la société industrielle se développent de plus en plus des sociétés d'information dans lesquelles le secteur de l'information n'est plus un service mais un secteur propre: Ce qui est, en outre visible dans l'augmentation des métiers de la communication et leurs représentants. Mais cela signifie aussi pour l'Eglise, à côté de toutes les chances, que toujours plus d'institutions de communication se disputent »'influence sociale« qui ne s'accroît pas à l'infini. Par la diversité de ces efforts de communication, l'Eglise dépasse, tout comme auparavant, toutes les autres grandes institutions de communication qui sont thématiquement limitées soit à l'économie et à la politique soit aux domaines culturels. En même temps apparait, comme problème élémentaire pour le présent et l'avenir des Eglises chrétiennes, la sauvegarde de son identité. Une certaine adaption à l'environnement sécularisé peut disparaître aux dépens d'une fonction spécifique et aussi, la plupart du temps, d'une intégration de l'Eglise. La séparation grandissante de la population nominalement chrétienne en activistes et en usufruitiers d'occasion met les représentants officiels de l'Eglise continuellement face à d'épineux conflits de but. Les Eglises devront ici spécifier leurs fonctions et définir leurs limites.

\section{RESUMEN}

Como criterio para medir el éxito de la Iglesia en su función como institución comunicadora se tiene en cuenta la medida en que ayuda a solucionar los problemas de las estructuras sociales de la colectividad y del individuo. En la situación de expansión mundial de los medios de comunicación social que vivirá la Iglesia en el año 2000 , resultan decisivas las estructuras de comunicación más relevantes, consideradas globalmente, y sus desarrollos, ó sea, los sistemas de comunicación en las democracias desarrolladas, en los estados comunistas relativamente desarrollados y en los países en desarrollo, comunistas ó no comunistas. La tendencia hacia la dictadura se agudizará en éstos últimos, la unidad en el bloque comunista seguirá difuminándose y las democracias desarrolladas apenas desmontarán sus peculiaridades nacionales bajo la influencia de acuerdos supranacionales. En las sociedades industriales se desarrollarán cada vez más instituciones en las que el sector informativo desaparece como servicio y se convierte en sector propio, lo cual se pone de manifiesto, por ejemplo, en la multiplicación de profesiones en el sector de la comunicación. Además de oportunidades, ello significa también para la Iglesia la multiplicación de instituciones que se disputan las limitadas formas de la ,influencia social“. En el terreno de la comunicación social la Iglesia contacta con todas las demás grandes instituciones de la difusión, que limitan su temática al campo de la economía, la política ó la cultura. Así aparece la salvaguardia de su identidad como el problema actual y futuro más elemental de la Iglesia. Es posible una cierta acomodación al entorno secular a costa de la función específica de la Iglesia y de su integración. La progresiva división de la población cristiana en activistas y usuarios ocasionales crea un conflicto permanente a la jerarquía. Las iglesias deberán especificar sus funciones y definir sus fronteras. 